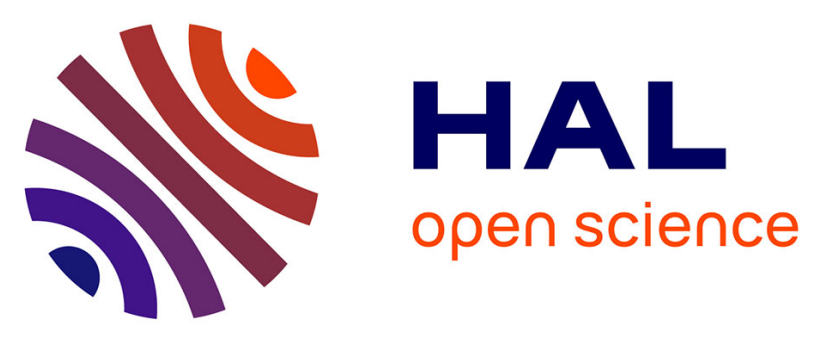

\title{
Competition between foraging predators and hiding preys as a nonzero-sum differential game
}

\author{
Andrei R. Akhmetzhanov, Pierre Bernhard, Frédéric Grognard, Ludovic
}

Mailleret

\section{To cite this version:}

Andrei R. Akhmetzhanov, Pierre Bernhard, Frédéric Grognard, Ludovic Mailleret. Competition between foraging predators and hiding preys as a nonzero-sum differential game. International Conference on Game Theory for Networks (GameNets 2009), May 2009, Istanbul, Turkey. pp.357 - 365, 10.1109/GAMENETS.2009.5137421 . hal-01091827

\section{HAL Id: hal-01091827 \\ https://hal.inria.fr/hal-01091827}

Submitted on 8 Dec 2014

HAL is a multi-disciplinary open access archive for the deposit and dissemination of scientific research documents, whether they are published or not. The documents may come from teaching and research institutions in France or abroad, or from public or private research centers.
L'archive ouverte pluridisciplinaire HAL, est destinée au dépôt et à la diffusion de documents scientifiques de niveau recherche, publiés ou non, émanant des établissements d'enseignement et de recherche français ou étrangers, des laboratoires publics ou privés.

\section{(ㅇ)(1) $\$$}

Distributed under a Creative Commons Attribution - NonCommercial| 4.0 International 


\title{
Competition between foraging predators and hiding preys as a nonzero-sum differential game
}

\author{
Andrei R. Akhmetzhanov, Pierre Bernhard, Frédéric Grognard and Ludovic Mailleret
}

\begin{abstract}
In this work we investigate a (seasonal) preypredator model where the system evolves during a season whose length is fixed. Predators have the choice between foraging the food (eating preys) and reproducing (laying eggs at a rate proportional to their energy). Preys can either eat, which would maintain their population in the absence of predators, or hide from the predators but they then suffer a positive mortality rate. In this case the population size can decrease even faster than if they were not hiding and were foraged by the predators. In their own turn they lay eggs at a constant rate whether they are hiding or eating. Following Darwin's principle that the fittest population will survive we postulate that both populations must maximize the number of their offspring, which yields a nonzerosum differential game.
\end{abstract}

\section{INTRODUCTION}

Prediction of the population dynamics plays a significant role in pest management and other fields of biological control, which is the use of predators or more generally natural enemies of pests in order to eradicate its population. Therefore we should predict the changes in the size of the pest population and describe how it evolves along the seasons. The formulation of the prey-predator model which will describe the relevant properties of the system and will be close to the experimental data obtained in fields is an important problem faced by mathematical biologists.

The most classical representation of these interactions are ordinary differential equations (e.g. Lotka-Volterra model) and difference equations (e.g. Nicholson-Bailey model). In our case we will study seasonal insects whose dynamics are continuous during the season and discrete between seasons. Such a system can not be described by the classical models recalled above [1]. In the literature, the classical approach is to consider explicitly the continuous and discrete dynamics, what produces a hybrid or more precisely impulsive model [2]. We follow the notations of [3] and call such models semi-dicrete. Indeed we suppose that the natural process is subdivided into separate seasons and periods of hibernation when all species die and the initial population for the next season is determined by the number of eggs produced by the species in the past.

The model presented in the paper describes the specific form of prey-predator interaction within a season. Both

A. R. Akhmetzhanov is with Institute for Problems in Mechanics of the Russian Academy of Sciences, Vernadsky Ave. 101-1, Moscow 119526, Russia akhmetzhanov@gmail.com

P. Bernhard and F. Grognard are with INRIA, Project COMORE, F-06902 Sophia Antipolis, France Pierre.Bernhardesophia.inria.fr Frederic.Grognardesophia.inria.fr

L. Mailleret is with INRA, UR880, F-06903 Sophia Antipolis, France Ludovic.Mailleretesophia.inra.fr populations of predators and preys are active and they can choose the appropriate tactic that gives the best response to the behavior of the opponent. Predators can either increase their energy by feeding on the preys or produce some offspring by laying eggs. However their reproduction rate depends on this reproductive energy which can be increased by feeding. If the preys can notice that the size of their population decreases very fast and it can become critically small, they can hide from the predators. But in this case they do not eat and their mortality rate becomes non-zero. We suppose also that the preys lay eggs at a constant rate all the time and it does not depend on their choice of the behavioral strategy either to hide or to eat. By eating they keep their mortality rate equal to zero. Such a model can be stated in terms of a nonzero-sum differential game such that both players (predators and preys) tend to maximize the amount of offspring they produce during a season. The work presented here actually follows a previous study in which the preys were not considered as capable of hiding and that reduced to an optimal control problem (one-player game) for the predators [4].

\section{MAIN MODEL}

\section{A. Formulation of a nonzero-sum differential game}

Let one consider a closed system of two species: predators and preys. The period of time when both populations are active is fixed at $T$. We will refer to $T$ as a season. We make use of two variables to describe the population of predators: the reproductive energy $p$ in average and the number of predators $z$. To describe the population density of preys we introduce the variable $n$. We suppose that both populations consist of two parts: a mature and an immature part. During the season, mature insects can invest in immatures by laying eggs. Between the seasons all matures die and immatures become matures for the next season. In this paper we are interested only in the within season dynamics.

At the beginning of the year all predators are small and $p$ equals to zero. Since predators are laying eggs with a rate proportional to the value of the energy $p$, it seems intuitive that most of them will try and forage the preys at the beginning and reproduce at the end, once they have gathered enough energy. We consider the case where the predator has a choice between feeding on the preys $(u=1)$ and laying eggs $(u=0)$. From the other side the preys have a choice either to hide $(v=0)$ or to eat $(v=1)$. Here the variables $u$ and $v$ play the role of the controls.

From the previous considerations and after rescaling the states and time so as to limit the number of parameters, we 
can then assume that the within season dynamics can be written in the form

$$
\dot{p}=-p+n u v, \quad \dot{n}=-\mu n(1-v)-n z u v
$$

where the parameter $\mu>0$ is the mortality rate of the preys. The value of $z$ is supposed to be constant during the season which is more or less relevant to the real situations in nature.

The number of offspring produced by the predators and preys along the season depends on the current size of the population

$$
J_{u}=\int_{0}^{T} p(t) z(1-u(t)) d t, \quad J_{v}=\int_{0}^{T} n(t) d t
$$

where the variable $z$ can be omitted as a constant.

The goal of the mathematical analysis is to define the optimal (or rational) strategy of the players and predict the actual behavior in real-life situations. A major step in the understanding of such game problems with several players has been done by John Nash. One says that the profile of strategies constitute a Nash equilibrium if: whenever one of the players deviates from his initial strategy he will not increase his own payoff. In the case of two players the pair of controls

$$
u=u^{*}(p, n, t), \quad v=v^{*}(p, n, t)
$$

is called a Nash equilibrium solution if the following inequalities hold

$$
\begin{gathered}
J_{u}\left(p, n, \tau, u, v^{*}\right) \leq J_{u}\left(p, n, \tau, u^{*}, v^{*}\right), \\
J_{v}\left(p, n, \tau, u^{*}, v\right) \leq J_{v}\left(p, n, \tau, u^{*}, v^{*}\right)
\end{gathered}
$$

for every alternative strategies $u$ and $v$. Let one introduce the value functions $\tilde{U}$ and $\tilde{V}$ for the predators and preys correspondingly. Roughly speaking the function $\tilde{U}(p, n, t)$ (or $\tilde{V}(p, n, t)$ ) give the equilibrium payoff expected by the player, if the game were to start at time $t$ in the state $(p(t), n(t))=(p, n)$. Assume that both value functions exist. Under regularity conditions (see [5, p. 292]), the functions $\tilde{U}$ and $\tilde{V}$ provide a solution to the system of Hamilton-JacobiBellman (HJB) equations

$$
\begin{aligned}
-\frac{\partial \tilde{U}}{\partial \tau}+\max _{0 \leq u \leq 1}\left[\frac{\partial \tilde{U}}{\partial p}\left(-p+n u v^{*}\right)+\right. \\
\left.\frac{\partial \tilde{U}}{\partial n}\left(-\mu\left(1-v^{*}\right) n-n z u v^{*}\right)+(1-u) p\right]=0 \\
-\frac{\partial \tilde{V}}{\partial \tau}+\max _{0 \leq v \leq 1}\left[\frac{\partial \tilde{V}}{\partial p}\left(-p+n u^{*} v\right)+\right. \\
\left.\frac{\partial \tilde{V}}{\partial n}\left(-\mu(1-v) n-n z u^{*} v\right)+n\right]=0
\end{aligned}
$$

where $u^{*}$ and $v^{*}$ provide the maximum in the other equation, $\tau$ is the reverse time, $\tau=T-t$. Regarding the terminal conditions

$$
\tilde{U}(p(T), n(T), T)=0, \quad \tilde{V}(p(T), n(T), T)=0
$$

\section{B. Transformation to $2 D$}

One can show that all the data are homogeneous of degree one in the state variables, and on the other hand, one of the state variables is always positive. This is a particular case of Noether's theorem in the calculus of variations about problems whose data is invariant under a group of transformations. Therefore we can reduce the phase space of the problem on degree one and study only the trandsformed system. To do so let one change the value function $\tilde{U}$ and $\tilde{V}$ to $\tilde{U}(p, n, \tau)=n U(x, \tau)$ and $\tilde{V}(p, n, \tau)=n V(x, \tau)$ where the new variable $x$ is introduced, $x=p / n$. In this case the system of HJB-equations transforms to

$$
\begin{array}{r}
h_{u} \triangleq-\pi_{\tau}+\max _{0 \leq u \leq 1}\left[\pi_{x}\left(-x\left(1-\mu\left(1-v^{*}\right)-z u v^{*}\right)+u v^{*}\right)+\right. \\
\left.U\left(-\mu(1-v)-z u v^{*}\right)+(1-u) x\right]=0 \\
h_{v} \triangleq-\nu_{\tau}+\max _{0 \leq v \leq 1}\left[\nu_{x}\left(-x\left(1-\mu(1-v)-z u^{*} v\right)+u^{*} v\right)+\right. \\
\left.V\left(-\mu(1-v)-z u^{*} v\right)+1\right]=0
\end{array}
$$

where we introduce the following notations for the conjugate variables $\pi_{x}=\partial U / \partial x$ and $\pi_{\tau}=\partial U / \partial \tau, \nu_{x}=\partial V / \partial x$ and $\nu_{\tau}=\partial V / \partial \tau$. The optimal behavior is the following

$$
u^{*}=\operatorname{Heav}\left(\mathcal{A}_{u}\right), \quad \mathcal{A}_{u}=\pi_{x}(x z+1) v-U v z-x
$$

$v^{*}=\operatorname{Heav}\left(\mathcal{A}_{v}\right), \quad \mathcal{A}_{v}=\nu_{x}(u(x z+1)-\mu x)+V(\mu-u z)$

We can see that only two variables $\tau$ and $x$ are left in the equations.

In order to solve the HJB equations, a characteristic system can be written as follows

$$
\begin{aligned}
x^{\prime} & =-\frac{\partial h_{u}}{\partial \pi_{x}}=x(1-\mu(1-v)-z u v)-u v, \\
\pi_{x}^{\prime} & =\frac{\partial h_{u}}{\partial x}+\frac{\partial h_{u}}{\partial U} \pi_{x}=-\pi_{x}+1-u, \\
\pi_{\tau}^{\prime} & =\frac{\partial h_{u}}{\partial \tau}+\frac{\partial h_{u}}{\partial U} \pi_{\tau}=-\pi_{\tau}(\mu(1-v)+z u v), \\
\nu_{x}^{\prime} & =\frac{\partial h_{v}}{\partial x}+\frac{\partial h_{v}}{\partial V} \nu_{x}=-\nu_{x}, \\
\nu_{\tau}^{\prime} & =\frac{\partial h_{v}}{\partial \tau}+\frac{\partial h_{v}}{\partial V} \nu_{\tau}=-\nu_{\tau}(\mu(1-v)+z u v), \\
U^{\prime} & =-\pi_{x} \frac{\partial h_{u}}{\partial \pi_{x}}-\pi_{\tau} \frac{\partial h_{u}}{\partial \pi_{\tau}}= \\
V^{\prime} & =-\nu_{x} \frac{\partial h_{v}}{\partial \nu_{x}}-\nu_{\tau} \frac{\partial h_{v}}{\partial \nu_{\tau}}=-V(\mu(1-v)+z u v)+1
\end{aligned}
$$

where the prime denotes the derivative w.r.t. reverse time.

If we know the values of $\pi_{i}, \nu_{i}(i=x, \tau)$, we can obtain the value functions $U$ and $V$ directly from the system of HJB-equations $h_{i}=0(i=u, v)$.

Terminal conditions at $t=T$ give $U(x, T)=0$ and $V(x, T)=0$. This leads to the equality $\pi_{x}(T)=\nu_{x}(T)=0$. From a biological point of view we can also state that $u(T)=0$ and $v(T)=1$ since it would not makes sense for the predators to try and increase their reproductive energy 
just before dying. They should consume their energy for reproduction $(u(T)=0)$ which allows the preys to safely eat $(v(T)=1)$. This can also be shown through mathematical considerations. Then

$$
\pi_{\tau}(T)=(1-u(T)) x(T)=x(T), \quad \nu_{\tau}(T)=1
$$

\section{PRIMARY SOLUTION}

\section{A. Appearance of the switching curve}

At $\tau=0$ we have

$$
\begin{array}{ll}
x^{\prime}=x, & \\
\pi_{x}^{\prime}=1-\pi_{x}, & \pi_{\tau}^{\prime}=0, \\
\nu_{x}^{\prime}=-\nu_{x}, & \nu_{\tau}^{\prime}=0, \\
U^{\prime}=x, & V^{\prime}=1
\end{array}
$$

or

$$
\begin{gathered}
x=x_{0} e^{\tau}, \quad \pi_{x}=1-e^{-\tau}, \quad \nu_{x}=0, \quad \pi_{\tau}=x_{0}, \quad \nu_{\tau}=1 \\
U=x-x_{0}, \quad V=\tau
\end{gathered}
$$

There is a switching curve $S_{1}$ that appears in the solution. The switching condition for the first player is the following $\mathcal{A}_{u}=\left(1-e^{-\tau}\right)(x z+1)-x\left(1-e^{-\tau}\right) z-x=\left(1-e^{-\tau}\right)-x=0$ Then

$$
S_{1}: \quad x=1-e^{-\tau}
$$

for $x \leq \frac{1}{2}$; indeed, the curve $x=1-e^{-\tau}$ can only be crossed by solutions if $x(T) \leq \frac{1}{4}$, which corresponds to an intersection at $x=\frac{1}{2}$.

First step of the construction of the characteristics is shown on Fig. 1. The blue line $S_{1}$ is the switching curve for the first player. Moving from the left side of $S_{1}$ to the right one the values $\pi_{x}$ and $\pi_{\tau}$ are changing smoothly. But the values of $\nu_{x}$ and $\nu_{\tau}$ have a jump, because the corresponding HJB-equation is changing. On the right side of it, the HJBequation has the form

$$
-\nu_{\tau}^{+}+\nu_{x}^{+}(1-x+x z)-V z+1=0
$$

where the + superscript denotes the values "after" the jump in reverse time.

A normal vector to the switching surface $S_{1}$ equals to

$$
\nu=\nabla S_{1}=\left(\partial S_{1} / \partial x, \partial S_{1} / \partial \tau\right)=(-1,1-x)
$$

Due to the continuity of the value function $\nabla V^{+}=\nabla V^{-}+$ $k \nu$ or

$$
\left(\begin{array}{c}
\nu_{x}^{+} \\
\nu_{\tau}^{+}
\end{array}\right)=\left(\begin{array}{c}
\nu_{x}^{-} \\
\nu_{\tau}^{-}
\end{array}\right)+k\left(\begin{array}{c}
-1 \\
1-x
\end{array}\right)=\left(\begin{array}{c}
-k \\
1+k(1-x)
\end{array}\right)
$$

with $\left(\nu_{x}^{-}, \nu_{t}^{-}\right)=(0,1)$. Substitution into the HJB-equation gives

$$
k=-\frac{\tau z}{2(1-x)+x z}
$$

Then

$$
\nu_{x}^{+}=\frac{\tau z}{2(1-x)+x z}, \quad \nu_{\tau}^{+}=1-\frac{(1-x) \tau z}{2(1-x)+x z}
$$

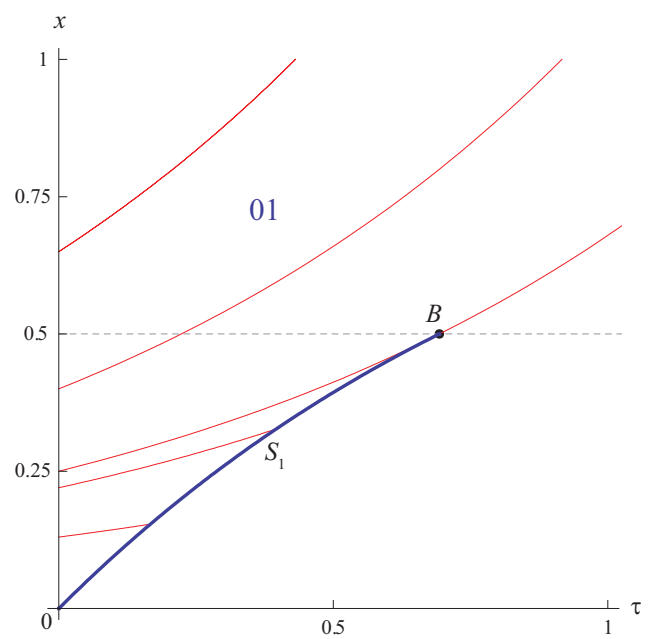

Fig. 1. Appearance of the switching curve $S_{1}$

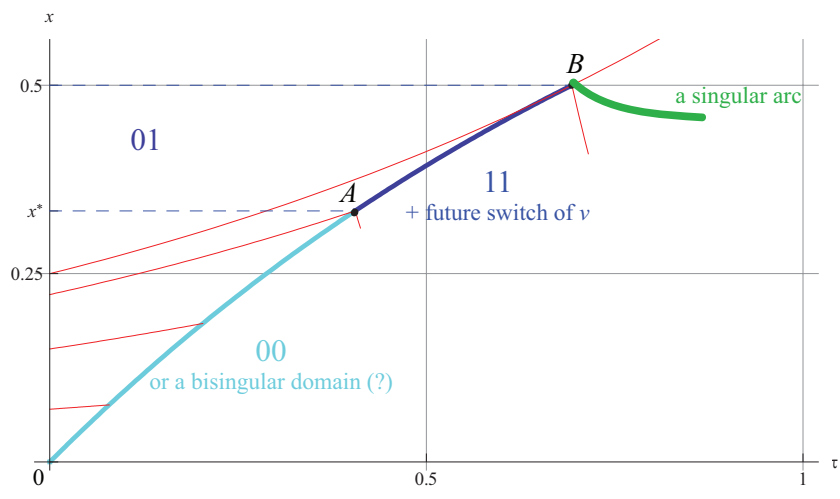

Fig. 2. Possible optimal behavior of the players after the switch on $S_{1}$

The argument in the Heaviside function of the second control $v$ becomes equal to

$$
\mathcal{A}_{v}^{+}=\frac{(2(1-x) \mu-(1-2 x) z) \tau}{2(1-x)+x z}
$$

The expression in the denominator $x z+2(1-x)$ is always positive since $x<1 / 2$ on $S_{1}$. Then $\mathcal{A}_{v}{ }^{+}>0$ if $2(1-x) \mu-$ $(1-2 x) z>0$ or $x>x^{*}=(z-2 \mu) / 2(z-\mu)$. This holds for all possible $x$ if $z<2 \mu$. Otherwise there is an internal point $x=x^{*}$ such that $\mathcal{A}_{v}{ }^{+}<0$ for $0 \leq x<x^{*}$ and $\mathcal{A}_{v}{ }^{+}>0$ for $x^{*}<x \leq 1 / 2$.

The following situation corresponding to the values $z=8$ and $\mu=2$ is shown on Fig. 2.

\section{B. Segment $A B$}

Suppose that the coordinate $x^{*}$ exists $\left(0<x^{*}<1 / 2\right)$ and consider the characteristic field emitted from the segment $A B$. In addition let's denote the coordinate of the switch on $S_{1}$ as $\left(x_{1}, \tau_{1}\right)$. Both controls equals to one (predators are feeding, preys continue to eat), $u\left(\tau_{1}+0\right)=v\left(\tau_{1}+0\right)=1$, 


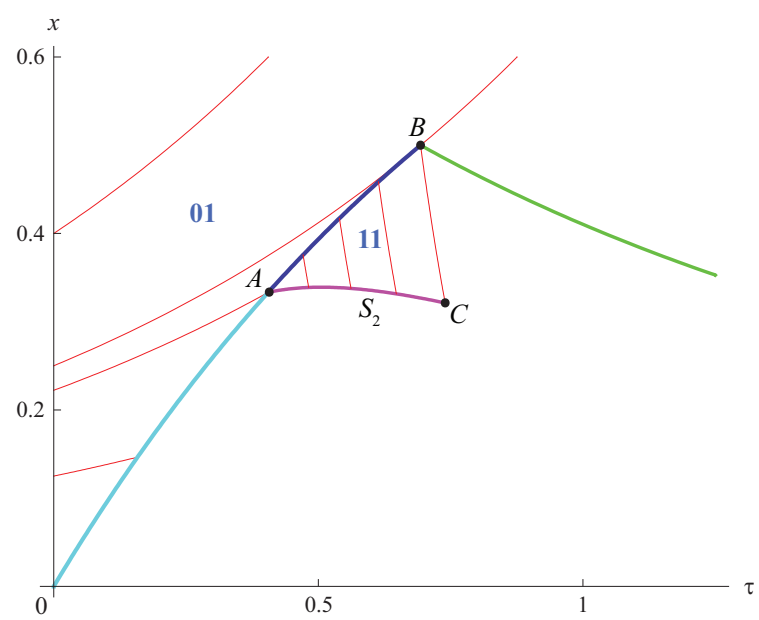

Fig. 3. The switching curve $S_{2}$

and the characteristic system is written in the form

$$
\begin{array}{ll}
x^{\prime}=-(z-1) x-1, & x\left(\tau_{1}\right)=x_{1}, \\
\pi_{x}^{\prime}=-\pi_{x}, & \pi_{x}\left(\tau_{1}\right)=1-e^{-\tau_{1}}=x_{1}, \\
\pi_{\tau}^{\prime}=-\pi_{\tau} z, & \pi_{\tau}\left(\tau_{1}\right)=x_{1} e^{-\tau_{1}}=x_{1}\left(1-x_{1}\right), \\
\nu_{x}^{\prime}=-\nu_{x}, & \nu_{x}\left(\tau_{1}\right)=\frac{\tau_{1} z}{2\left(1-x_{1}\right)+x_{1} z}, \\
\nu_{\tau}^{\prime}=-\nu_{\tau} z, & \nu_{\tau}\left(\tau_{1}\right)=1-\frac{\left(1-x_{1}\right) \tau_{1} z}{2\left(1-x_{1}\right)+x_{1} z}, \\
U^{\prime}=-U z, & U\left(\tau_{1}\right)=x_{1}\left(1-e^{-\tau_{1}}\right)=x_{1}^{2}, \\
V^{\prime}=-V z+1, & V\left(\tau_{1}\right)=\tau_{1}
\end{array}
$$

Then

$$
\begin{gathered}
x=x_{1} e^{-(z-1)\left(\tau-\tau_{1}\right)}-\frac{1-e^{-(z-1)\left(\tau-\tau_{1}\right)}}{z-1} \\
\pi_{x}=x_{1} e^{-\left(\tau-\tau_{1}\right)}, \quad \pi_{\tau}=x_{1}\left(1-x_{1}\right) e^{-z\left(\tau-\tau_{1}\right)} \\
\nu_{x}=\nu_{x}\left(\tau_{1}\right) e^{-\left(\tau-\tau_{1}\right)}, \quad \nu_{\tau}=\nu_{\tau}\left(\tau_{1}\right) e^{-z\left(\tau-\tau_{1}\right)} \\
U=x_{1}^{2} e^{-z\left(\tau-\tau_{1}\right)}, \quad V=\tau_{1} e^{-z\left(\tau-\tau_{1}\right)}+\frac{1-e^{-z\left(\tau-\tau_{1}\right)}}{z}
\end{gathered}
$$

The switching surface for the second player can be obtained from the equality

$$
\mathcal{A}_{v}=\nu_{x}(1+x(z-\mu))-V(z-\mu)=0
$$

or

$$
\begin{aligned}
S_{2}: & \frac{\tau_{1} z e^{-\left(\tau-\tau_{1}\right)}}{2\left(1-x_{1}\right)+x_{1} z}(1+x(z-\mu)) \\
& -\left(\tau_{1} e^{-z\left(\tau-\tau_{1}\right)}+\frac{1-e^{-z\left(\tau-\tau_{1}\right)}}{z}\right)(z-\mu)=0
\end{aligned}
$$

where

$$
x=x_{1} e^{-(z-1)\left(\tau-\tau_{1}\right)}-\frac{1-e^{-(z-1)\left(\tau-\tau_{1}\right)}}{z-1}, \quad x_{1}=1-e^{-\tau_{1}}
$$

Finally, we have the situation shown on Fig. 3.

\section{A singular arc}

Since the unchanged continuation of the terminal solution that is tangent to $S_{1}$ and solutions that lead to $S_{2}$ leave an empty space between them, there must exist a singular arc $S^{\sigma}$ that is emitted from this point along which the first player applies the intermediate control $u=u^{\sigma}$ while the control of the second player remains the same $v=1$. To define such a curve $S^{\sigma}$ let one write the necessary conditions of its existence

$$
\begin{gathered}
h_{u}=-\pi_{\tau}+x\left(1-\pi_{x}\right)=0 \\
\mathcal{A}_{u}=\pi_{x}(x z+1)-U z-x=0, \quad \mathcal{A}_{u}{ }^{\prime}=0
\end{gathered}
$$

We have

$$
\begin{aligned}
& \mathcal{A}_{u}{ }^{\prime}=\left\{\mathcal{A}_{u} h_{u}\right\}= \\
& \frac{\partial h_{u}}{\partial \pi_{x}}\left(\frac{\partial \mathcal{A}_{u}}{\partial x}+\pi_{x} \frac{\partial \mathcal{A}_{u}}{\partial U}\right)+\pi_{\tau}\left(\frac{\partial \mathcal{A}_{u}}{\partial \tau}+\frac{\partial h_{u}}{\partial \pi_{\tau}} \frac{\partial \mathcal{A}_{u}}{\partial U}\right)- \\
& \frac{\partial \mathcal{A}_{u}}{\partial \pi_{x}}\left(\frac{\partial h_{u}}{\partial x}+\pi_{x} \frac{\partial h_{u}}{\partial U}\right)-\frac{\partial \mathcal{A}_{u}}{\partial \pi_{\tau}}\left(\frac{\partial h_{u}}{\partial \tau}+\pi_{\tau} \frac{\partial h_{u}}{\partial U}\right)= \\
& \quad-1+x\left(1+z\left(\pi_{x}-1\right)\right)+\pi_{x}+z \pi_{\tau}=0
\end{aligned}
$$

where the braces $\{\cdot\}$ denote the Jacobi (Poisson) brackets. Then the conjugate variables are expressed as follows

$$
\pi_{x}=1-x, \quad \pi_{\tau}=x(1-x)
$$

The value function of the first player

$$
U=x(1-x)+\frac{1-2 x}{z}
$$

The expression for the singular control $u^{\sigma}$ can be obtained using the equality of the second derivative of $\mathcal{A}_{u}$ to zero

$$
\begin{aligned}
\mathcal{A}_{u}{ }^{\prime \prime}=\left\{\left\{\mathcal{A}_{u} h_{u}\right\} h_{u}\right\}=\left\{\left\{\left(\pi_{x}-1+x\right) h_{u}\right\} h_{u}\right\}= \\
-2 x+u^{\sigma}(x z+2)=0
\end{aligned}
$$

Then

$$
u^{\sigma}=\frac{2 x}{x z+2}
$$

We have also

$$
x^{\prime}=-\frac{x^{2} z}{x z+2}, \quad x\left(\tilde{\tau}_{1}\right)=\frac{1}{2}, \quad \tilde{\tau}_{1}=\ln 2
$$

Finally we can integrate this differential equation with the given initial conditions and obtain the following expression for the singular arc

$$
S^{\sigma}: \quad-\ln x+\frac{2}{x z}-\frac{4}{z}=\tau, \quad \tau \geq \tilde{\tau}_{1}
$$

Consider the moment of switching from $u=u^{\sigma}$ to $u=1$ at some point of the singular $\operatorname{arc}\left(x^{\sigma}, \tau^{\sigma}\right)$. The HJB-equation is changed to the following equation

$$
-\nu_{\tau}^{\sigma}+\nu_{x}^{\sigma}(1-x+x z)-V z+1=0
$$

and there is a jump in the costate variables of the second player. 
Along the singular arc $S^{\sigma}$ :

$$
\begin{aligned}
V^{\prime}=1-V z u^{\sigma}=1-V \frac{2 x z}{x z+2}, \quad x^{\prime}=-\frac{x^{2} z}{x z+2}, \\
V(\ln 2)=\ln 2, \quad x(\ln 2)=\frac{1}{2}
\end{aligned}
$$

This can be rewritten in the form

$$
\frac{d V}{d x}=\frac{2 V x z-x z-2}{x^{2} z}, \quad V(1 / 2)=\ln 2
$$

Therefore

$V=4 x^{2} \ln 2+\frac{(1-2 x)\left(4+8 x+16 x^{2}+3 x z(1+2 x)\right)}{6 x z} \triangleq V^{\sigma}$

Choose the variable $s=x$ as a parameter of the singular arc. In this case

$$
\frac{\partial \tau}{\partial s}=-\frac{1}{x}-\frac{2}{x^{2} z}
$$

and

$\frac{\partial V}{\partial s}=-\frac{2+4 x^{3}(8-3 z(2 \ln 2-1))}{3 x^{2} z}=\nu_{x}^{\sigma}-\nu_{\tau}^{\sigma}\left(\frac{1}{x}+\frac{4}{x^{2} z}\right)$

After the substitution to the changed HJB-equation we have the following values of conjugate variables

$$
\begin{aligned}
& \nu_{\tau}^{\sigma}=\frac{2+3 x z+4 x^{3}(8-3 z(2 \ln 2-1))}{6(x z+1)}, \\
& \nu_{x}^{\sigma}=\frac{4+3 x z-4 x^{3}(8-3 z(2 \ln 2-1))}{6 x(x z+1)}
\end{aligned}
$$

These values are the initial conditions of characteristic trajectories emitted from the singular curve $S^{\sigma}$ in the domain 11. For this domain we have again

$$
\begin{array}{ll}
x^{\prime}=-(z-1) x-1, & x\left(\tau^{\sigma}\right)=x^{\sigma}, \\
\pi_{x}^{\prime}=-\pi_{x}, & \pi_{x}\left(\tau^{\sigma}\right)=1-x^{\sigma}, \\
\pi_{\tau}^{\prime}=-\pi_{\tau} z, & \pi_{\tau}\left(\tau^{\sigma}\right)=x^{\sigma}\left(1-x^{\sigma}\right), \\
\nu_{x}^{\prime}=-\nu_{x}, & \nu_{x}\left(\tau^{\sigma}\right)=\nu_{x}^{\sigma}, \\
\nu_{\tau}^{\prime}=-\nu_{\tau} z, & \nu_{\tau}\left(\tau^{\sigma}\right)=\nu_{\tau}^{\sigma}, \\
U^{\prime}=-U z, & U\left(\tau^{\sigma}\right)=x^{\sigma}\left(1-x^{\sigma}\right)+\frac{1-2 x^{\sigma}}{z}, \\
V^{\prime}=-V z+1, & V\left(\tau^{\sigma}\right)=V^{\sigma}
\end{array}
$$

The switching condition

$$
\mathcal{A}_{v}=\nu_{x}(1+x(z-\mu))-V(z-\mu)=0
$$

or

$$
\begin{array}{ll}
S_{2}^{\sigma}: \quad \nu_{x}^{\sigma} e^{-\left(\tau-\tau^{\sigma}\right)}(1+x(z-\mu))- \\
& \left(V^{\sigma} e^{-z\left(\tau-\tau^{\sigma}\right)}+\frac{1-e^{-z\left(\tau-\tau^{\sigma}\right)}}{z}\right)(z-\mu)=0
\end{array}
$$

where

$$
x=x^{\sigma} e^{-(z-1)\left(\tau_{2}-\tau^{\sigma}\right)}-\frac{1-e^{-(z-1)\left(\tau_{2}-\tau^{\sigma}\right)}}{z-1}
$$

This curve is indicated on Fig. 4 by the segment $C D$.

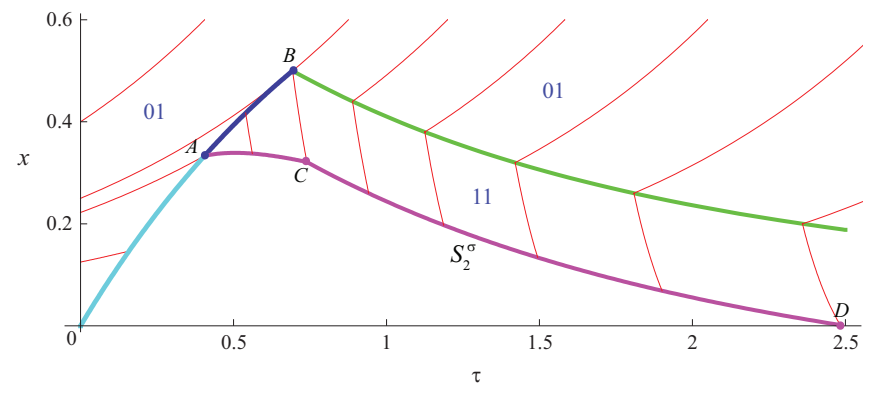

Fig. 4. Construction of the switching curve $S_{2}^{\sigma}$

\section{BI-SINGULAR SOLUTION}

\section{A. Derivation of the Hamilton-Jacobi (HJ) equations}

A simple Nash argument shows that it is not possible to have the region 00 inside the domain $0 A C D$ (see Fig. 4). Indeed, let us consider the segment $0 A$; in forward time, a solution initiated on the right of $0 A$ with the controls $(0,0)$ would be such that the predators only reproduce until the final time, while the preys first hide until reaching $0 A$ and then eat until the final time. Obviously, the preys can improve their situation from there by instead choosing to eat all the time, since they are not threatened by the preadtors, which are reproducing. The controls $(0,0)$ can therefore not be part of a Nash solution on the right of $0 A$. Further analysis of $A_{u}^{+}$and $A_{v}^{+}$shows that no solution with bang-bang control values can be emitted from $0 A$.

The phenomenon of the appearance of the bi-singular solution for the class of nonzero-sum differential games has been recently investigated [6]. To define necessary equations for the value function in the bi-singular domain let one eliminate the controls $v$ and $u$ from the pairs of the equations

$$
\begin{gathered}
h_{u}=-\pi_{\tau}-\pi_{x} x(1-\mu(1-v))-\mu U(1-v)+x=0, \\
\mathcal{A}_{u}=\pi_{x}(x z+1) v-U v z-x=0 \\
h_{v}=-\nu_{\tau}-\nu_{x} x(1-\mu)-\mu V+1=0, \\
\mathcal{A}_{v}=\nu_{x}(u(x z+1)-\mu x)+V(\mu-u z)=0
\end{gathered}
$$

This leads to the following PDEs which are denoted HJequations (instead of the previous HJB-equations):

$$
\begin{gathered}
\hat{h}_{u} \triangleq-\pi_{\tau}-\pi_{x} x(1-\mu)-\mu U+x-\frac{\mu x\left(\pi_{x} x-U\right)}{\pi_{x}(x z+1)-U z}=0 \\
\hat{h}_{v} \triangleq-\nu_{\tau}-\nu_{x} x(1-\mu)-\mu V+1=0
\end{gathered}
$$

The bi-singular controls can be obtained from the solution of these PDEs

$$
u^{\sigma}=\frac{\mu\left(\nu_{x} x-V\right)}{\nu_{x}(x z+1)-V z}, \quad v^{\sigma}=\frac{x}{\pi_{x}(x z+1)-U z}
$$

\section{B. Solution of the second HJ-equation}

Consider the HJ-equation regarding the second player

$$
\hat{h}_{v}=-\nu_{\tau}+\nu_{x} x(\mu-1)-\mu V+1=0
$$


with singular control

$$
u^{\sigma}=\frac{\mu\left(\nu_{x} x-V\right)}{\nu_{x}(x z+1)-V z} \quad\left(0 \leq u^{\sigma} \leq 1\right)
$$

The Characteristic system has the form

$$
\begin{aligned}
& x^{\prime}=-\frac{\partial \hat{h}_{v}}{\partial \nu_{x}}=-x(\mu-1), \\
& \nu_{x}^{\prime}=\frac{\partial \hat{h}_{v}}{\partial x}+\nu_{x} \frac{\partial \hat{h}_{v}}{\partial V}=-\nu_{x}, \\
& \nu_{\tau}^{\prime}=\frac{\partial \hat{h}_{v}}{\partial \tau}+\nu_{\tau} \frac{\partial \hat{h}_{v}}{\partial V}=-\mu \nu_{\tau}, \\
& V^{\prime}=-\nu_{x} \frac{\partial \hat{h}_{v}}{\partial \nu_{x}}-\nu_{\tau} \frac{\partial \hat{h}_{v}}{\partial \nu_{\tau}}=-\mu V+1, \\
& x\left(\tau_{1}\right)=x_{1}, \quad V\left(\tau_{1}\right)=\tau_{1}
\end{aligned}
$$

If we take the segment $0 A$ as the boundary we need to recalculate the values of the costate variables $\nu_{x}\left(\tau_{1}\right)$ and $\nu_{\tau}\left(\tau_{1}\right):$

$$
\left(\begin{array}{l}
\nu_{x}\left(\tau_{1}\right) \\
\nu_{\tau}\left(\tau_{1}\right)
\end{array}\right)=\left(\begin{array}{c}
-k \\
1+k(1-x)
\end{array}\right)
$$

regarding the $\mathrm{HJ}$-equation

$$
-\nu_{\tau}\left(\tau_{1}\right)+\nu_{x}\left(\tau_{1}\right)(\mu-1) x_{1}-\mu \tau_{1}+1=0
$$

Then

$$
k=-\frac{\mu \tau_{1}}{\mu x_{1}+1-2 x_{1}}
$$

and

$$
\nu_{x}\left(\tau_{1}\right)=\frac{\mu \tau_{1}}{\mu x_{1}+1-2 x_{1}}, \quad \nu_{\tau}\left(\tau_{1}\right)=1-\frac{\left(1-x_{1}\right) \mu \tau_{1}}{\mu x_{1}+1-2 x_{1}}
$$

Then

$$
\begin{gathered}
x=x_{1} e^{-(\mu-1)\left(\tau-\tau_{1}\right)}, \\
\nu_{x}=\nu_{x}\left(\tau_{1}\right) e^{-\left(\tau-\tau_{1}\right)}, \quad \nu_{\tau}=\nu_{\tau}\left(\tau_{1}\right) e^{-\mu\left(\tau-\tau_{1}\right)}, \\
V=\tau_{1} e^{-\mu\left(\tau-\tau_{1}\right)}+\frac{1-e^{-\mu\left(\tau-\tau_{1}\right)}}{\mu}
\end{gathered}
$$

By substitution of this solution we can write the expression for the singular control $u^{\sigma}$. On the segment $0 A$ it equals to

$$
u^{\sigma}\left(\tau_{1}\right)=\frac{\mu \nu_{x}\left(\tau_{1}\right) x_{1}-\mu \tau_{1}}{\nu_{x}\left(\tau_{1}\right)\left(x_{1} z+1\right)-\tau_{1} z}=\frac{\mu\left(1-2 x_{1}\right)}{\left(1-2 x_{1}\right) z-\mu}
$$

which belongs to the interval between zero and one. The singular control equals exactly one at the boundary point $A$ :

$$
\left.u^{\sigma}\left(\tau_{1}\right)\right|_{x_{1}=x^{*}}=1
$$

The result is shown on Fig. 5. The horizontal line for $x=0$ is at level $u^{\sigma}=\mu / z$.

Now let's take the segment $A C$ into account. If we denote the coordinates of the point on $A C$ as $\left(x_{2}, \tau_{2}\right) \in S_{2}$ and corresponding to it the point on $A B$ as $\left(x_{1}, \tau_{1}\right)$ we can write

$$
\begin{gathered}
x_{2}=x_{1} e^{-(z-1)\left(\tau_{2}-\tau_{1}\right)}-\frac{1-e^{-(z-1)\left(\tau_{2}-\tau_{1}\right)}}{z-1} \\
\nu_{x}\left(\tau_{2}\right)=\frac{\tau_{1} z e^{-\left(\tau_{2}-\tau_{1}\right)}}{2\left(1-x_{1}\right)+x_{1} z},
\end{gathered}
$$

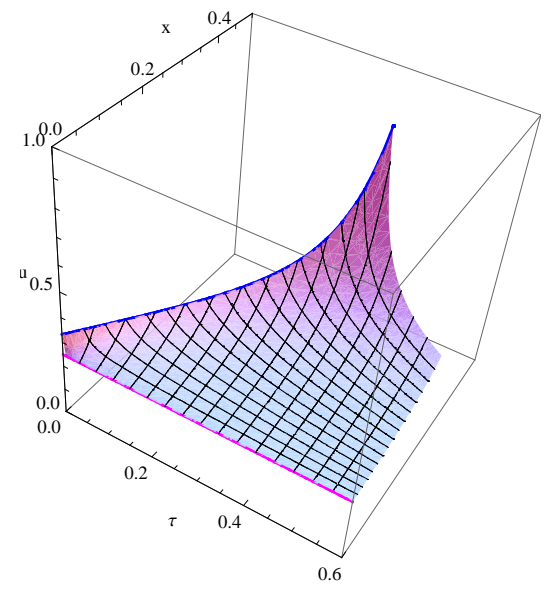

Fig. 5. Solution of the 2 nd HJ-equation with a boundary at $0 A$

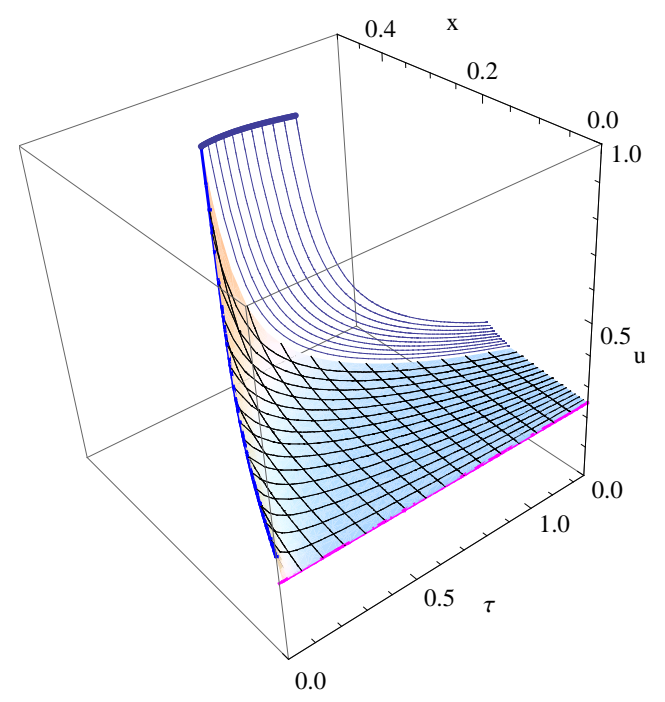

Fig. 6. Solution of the 2 nd HJ-equation with a boundary at $0 A C$

$$
\begin{gathered}
\nu_{\tau}\left(\tau_{2}\right)=\left(1-\frac{\left(1-x_{1}\right) \tau_{1} z}{2\left(1-x_{1}\right)+x_{1} z}\right) e^{-z\left(\tau_{2}-\tau_{1}\right)} \\
V\left(\tau_{2}\right)=\tau_{1} e^{-z\left(\tau_{2}-\tau_{1}\right)}+\frac{1-e^{-z\left(\tau_{2}-\tau_{1}\right)}}{z}
\end{gathered}
$$

with $u^{\sigma}\left(\tau_{2}\right)=1$. Then

$$
\begin{gathered}
x=x_{2} e^{-(\mu-1)\left(\tau-\tau_{2}\right)} \\
\nu_{x}=\nu_{x}\left(\tau_{2}\right) e^{-\left(\tau-\tau_{2}\right)}, \quad \nu_{\tau}=\nu_{\tau}\left(\tau_{2}\right) e^{-\mu\left(\tau-\tau_{2}\right)} \\
V=V\left(\tau_{2}\right) e^{-\mu\left(\tau-\tau_{2}\right)}+\frac{1-e^{-\mu\left(\tau-\tau_{2}\right)}}{\mu}
\end{gathered}
$$

Using these expressions we can obtain the value of the singular control $u^{\sigma}$. The solution is shown on Fig. 6 by the set of blue curves started from the segment $A C$. The surface closer to us indicates the previous solution.

The characteristics of the bi-singular field (which are not the game trajectories) intersect the field (11) coming, in backward time, from the singular arc. Therefore we can construct the singular line of the dispersal type such that $V_{\sigma}=V_{11}$ on it, where the value $V_{\sigma}$ denotes the solution 


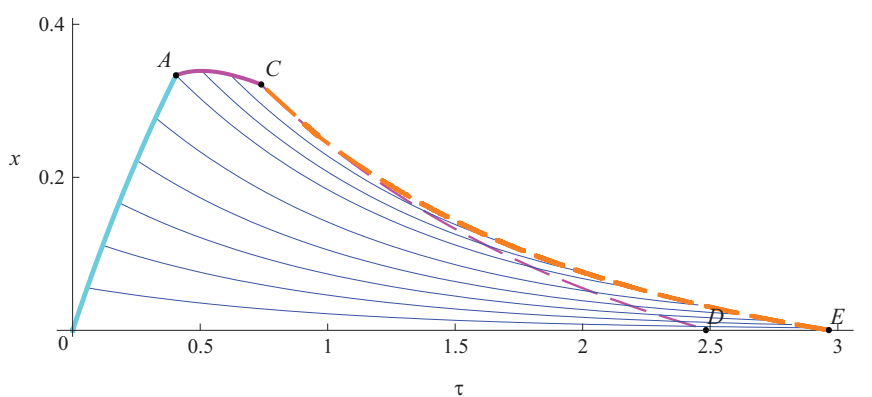

Fig. 7. Final construction of the characteristic field for the 2 nd HJ-equation

in the bi-singular domain, $V_{11}-$ in the domain (11). This gives the following construction shown on Fig. 7, where the dispersal line is indicated by the segment $C E$. The details of its construction are presented in the following subsection.

\section{Construction of the dispersal line $C E$}

For one part of $C E$ we should compare characteristics emitted from $0 A$ and characteristics emitted from the singular arc. They will give a lower part of $C E$. For another part we should consider the field (11) and bi-singular characteristics emitted from segment $A C$.

For the characteristics from $0 A$ we have

$x_{11}=x_{1} e^{-(\mu-1)\left(\tau-\tau_{1}\right)}, \quad V_{11}=\tau_{1} e^{-\mu\left(\tau-\tau_{1}\right)}+\frac{1-e^{-\mu\left(\tau-\tau_{1}\right)}}{\mu}$

for the characteristics from $A C$

$$
\begin{gathered}
x_{11}=x_{2} e^{-(\mu-1)\left(\tau-\tau_{2}\right)} \\
V_{11}=\left(\tau_{1} e^{-z\left(\tau_{2}-\tau_{1}\right)}+\frac{1-e^{-z\left(\tau_{2}-\tau_{1}\right)}}{z}\right) e^{-\mu\left(\tau-\tau_{2}\right)}+ \\
\frac{1-e^{-\mu\left(\tau-\tau_{2}\right)}}{\mu}
\end{gathered}
$$

where $\left(\tau_{1}, x_{1}\right) \in 0 B$ and $\left(\tau_{2}, x_{2}\right) \in A C$.

If we consider a primary domain we can write

$$
\begin{gathered}
x_{\sigma}=x^{\sigma} e^{-(z-1)\left(\tau-\tau^{\sigma}\right)}-\frac{1-e^{-(z-1)\left(\tau-\tau^{\sigma}\right)}}{z-1}, \\
V_{\sigma}=\frac{1-e^{-z\left(\tau-\tau^{\sigma}\right)}}{z}+e^{-z\left(\tau-\tau^{\sigma}\right)}\left(4\left(x^{\sigma}\right)^{2} \ln 2+\right. \\
\left.\frac{\left(1-2 x^{\sigma}\right)\left(4+8 x^{\sigma}+16\left(x^{\sigma}\right)^{2}+3 x^{\sigma} z\left(1+2 x^{\sigma}\right)\right)}{6 x^{\sigma} z}\right)
\end{gathered}
$$

where the coordinates $\left(x^{\sigma}, \tau^{\sigma}\right)$ belong to the singular arc $S^{\sigma}$.

The coordinates $\left(\tau_{3}, x_{3}\right)$ of the point on $C F$ can be obtained through equations

$$
\left.V_{11}\right|_{\tau=\tau_{3}}=\left.V_{\sigma}\right|_{\tau=\tau_{3}} \text { and }\left.x_{11}\right|_{\tau=\tau_{3}}=\left.x_{\sigma}\right|_{\tau=\tau_{3}}
$$

\section{Solution of the first HJ-equation}

Consider the following PDE

$$
\begin{aligned}
\hat{h}_{u}=-\pi_{\tau}+ & x\left(1-\pi_{x}\right)+ \\
\mu\left(\pi_{x} x-U\right) & \left(1-\frac{x}{\pi_{x}(x z+1)-U z}\right)=0
\end{aligned}
$$

with respect to singular control in the form

$$
v^{\sigma}=\frac{x}{\pi_{x}(x z+1)-U z}
$$

The corresponding characteristic system can be written analogously to the previous case

$$
\begin{aligned}
& x^{\prime}=-(\mu-1) x+\frac{\mu x U}{\left(\pi_{x}(x z+1)-U z\right)^{2}}, \\
& \pi_{x}^{\prime}=1-\pi_{x}-\frac{\mu\left(\pi_{x} x-U\right)}{\pi_{x}(x z+1)-U z}, \\
& \pi_{\tau}^{\prime}=-\mu \pi_{\tau}\left(1-\frac{\pi_{x} x}{\left(\pi_{x}(x z+1)-U z\right)^{2}}\right), \\
& U^{\prime}=x-\mu U-\frac{\mu x\left(U^{2} z+\pi_{x}^{2} x(x z+1)-2 U \pi_{x}(x z+1)\right)}{\left(\pi_{x}(x z+1)-U z\right)^{2}}
\end{aligned}
$$

with initial condition on the segment $0 A$

$x\left(\tau_{1}\right)=x_{1}, \pi_{\tau}\left(\tau_{1}\right)=x_{1}\left(1-x_{1}\right), \pi_{x}\left(\tau_{1}\right)=x_{1}, U\left(\tau_{1}\right)=x_{1}^{2}$

Notice that

$$
\left(\frac{\partial \tilde{U}}{\partial n}\right)^{\prime}=\left(\pi_{x} x-U\right)^{\prime}=-\mu\left(\pi_{x} x-U\right)
$$

But in our case $\left.\left(\pi_{x} x-U\right)\right|_{\tau=\tau_{1}}=0$. Therefore $\pi_{x} x-U=$ 0 all the time. This leads to the characteristic equation for $\pi_{x}$ of the form $\pi_{x}^{\prime}=1-\pi_{x}$ with initial condition on $0 A$ : $\alpha\left(\tau_{1}\right)=1-e^{-\tau_{1}}=x_{1}$. Then $\pi_{x}=1-e^{-\tau}$ and the singular control can be written in the form directly depending on $x$ and $\tau$ :

$$
v^{\sigma}=\frac{x}{1-e^{-\tau}}
$$

For the characteristic field emitted for the segment $A C$ the situation is more complicated since the conjugate variables have a jump on it. Suppose that the segment $S_{2}$ is defined through the following conditions

$$
\begin{gathered}
S_{2}: \quad \frac{\tau_{1} z e^{-\left(\tau-\tau_{1}\right)}}{2\left(1-x_{1}\right)+x_{1} z}(1+x(z-\mu)) \\
-\left(\tau_{1} e^{-z\left(\tau-\tau_{1}\right)}+\frac{1-e^{-z\left(\tau-\tau_{1}\right)}}{z}\right)(z-\mu)=0 \\
x=x_{1} e^{-(z-1)\left(\tau-\tau_{1}\right)}-\frac{1-e^{-(z-1)\left(\tau-\tau_{1}\right)}}{z-1}\left(x_{1}=1-e^{-\tau_{1}}\right)
\end{gathered}
$$

Suppose that $\tau_{1}=\tau_{1}(x, \tau)$. If we differentiate the last equation for $x$ we obtain

$$
\begin{aligned}
& \frac{\partial \tau_{1}}{\partial x}=\frac{1-x_{1}+x_{1} z}{(1-x+x z)\left(2\left(1-x_{1}\right)+x_{1} z\right)} \\
& \frac{\partial \tau_{1}}{\partial \tau}=\frac{1-x_{1}+x_{1} z}{2\left(1-x_{1}\right)+x_{1} z}
\end{aligned}
$$




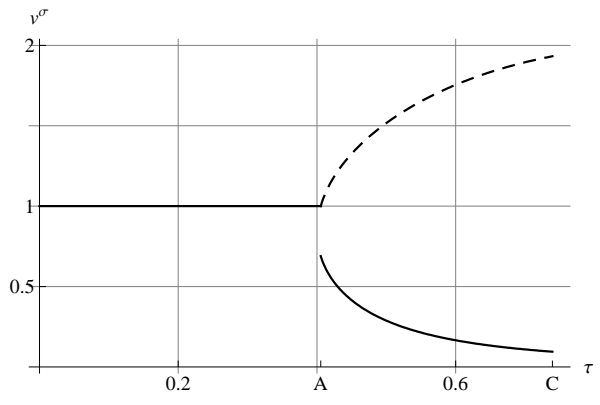

Fig. 8. Singular control $v^{\sigma}$ on the boundary of the bi-singular domain

To calculate the normal vector to $S_{2}$ we need to calculate the gradient

$$
\nabla S_{2}=\left(\partial S_{2} / \partial x, \partial S_{2} / \partial \tau\right)
$$

Then we can derive the expressions for $\partial S_{2} / \partial x$ and $\partial S_{2} / \partial \tau$ but we will omit this part since they have too complicated a form. Taking into account that

$\pi_{x}^{+}=x_{1} e^{-\left(\tau-\tau_{1}\right)}+k \frac{\partial S_{2}}{\partial x}, \pi_{\tau}^{+}=x_{1}\left(1-x_{1}\right) e^{-z\left(\tau-\tau_{1}\right)}+k \frac{\partial S_{2}}{\partial \tau}$

we can substitute these values into the HJ-equation

$-\pi_{\tau}^{+}+x\left(1-\pi_{x}^{+}\right)+\mu\left(\pi_{x}^{+} x-U\right)\left(1-\frac{x}{\pi_{x}^{+}(x z+1)-U z}\right)=0$

with $U=x_{1}^{2} e^{-z\left(\tau-\tau_{1}\right)}$ and obtain the equation w.r.t. the scalar $k$. After that, we can write the new values of the conjugate variables $\pi_{x}^{+}$and $\pi_{\tau}^{+}$.

We should notice that there are two possible solutions $k_{1}$ and $k_{2}$. The value of $k_{1}$ is negative on $A C$ and equals to zero at point $A$. But this branch corresponds to the situation when $v^{\sigma} \geq 1$. Another branch of the solution with $k_{2}>0$ corresponds to the values $v^{\sigma} \in(0,1)$, see Fig. 8 .

The resulting characteristic field for the segment $0 A$ and $A C$ is shown on Fig. 9.

\section{Construction of the real game trajectories}

Using the constructed solution of the decomposed system of two HJ-equations, we can compute the pair of singular controls $u^{\sigma}$ and $v^{\sigma}$ in the domain $0 A C E$. Thus, we can construct the real game trajectories in the bi-singular domain. They are shown on Fig. 10. We can identify our main difficulty on this figure. Even though the whole space was filled with the characteristics for the decomposed system of two HJ equations, there is an empty subdomain in the final portrait of optimal trajectories (shaded area of Figure 10). This is a strong indication that some important things may have been missed in the process of deriving the solution.

\section{CONCLUSIONS}

We did not finalize the solution and did not cover the whole phase space by the field of characteristics. But our work is still in progress and we will continue our research. Nevertheless, we would like to point out that in the paper we investigated the transition from bang-bang controls to bisingular ones and apply the method of characteristics to a

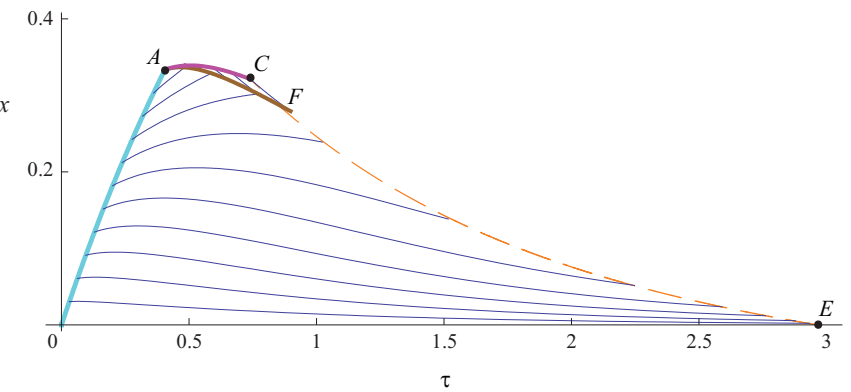

Fig. 9. Characteristic field for the first HJ-equation

system of two HJB-equations. Although the fact that we did not find the solution in a subdomain of the phase space can be crucial and a new idea of the solution can change the whole picture drastically we think that the proposed solution will remain fulfilled in a sufficiently large area of the phase space after.

The theory of nonzero-sum differential games is not welldeveloped yet. There are no theorems of existence and uniqueness of their solution [6], [7], [8] so that it might be possible that no Nash equilibrium exist from the initial states in the "void" zone. There might also be several, may be an infinity, of Nash equilibria. There is no strict classification of possible singular surfaces as one has been done for zero-sum differential games, see for example [9, p. 121]. Therefore this work is only an attempt to study a particular nonzero-sum differential games with an application in behavioral ecology.

\section{ACKNOWLEDGMENTS}

This research has been supported by the 'Lutins\&Co' COLOR project of INRIA Sophia Antipolis-Méditerranée and the 'IA2L' project of the SPE department of INRA. The work of the first author has been partially supported by the Russian Foundation for Basic Research (grant 07-04-00418).

\section{REFERENCES}

[1] S.A.H. Geritz and E. Kisdi, On the mechanistic underpinning of discrete-time population models with complex dynamics, Journal of Theoretical Biology, vol. 228, 2004, pp 261-269.

[2] W. W. Murdoch, C. J. Briggs and R. M. Nisbet, Consumer-resource dynamics., Princeton University Press, Princeton, NJ; 2003.

[3] E. Pachepsky, R. M. Nisbet and W. W. Murdoch, Between discrete and continuous consumer-resource dynamics with synchronized reproduction, Ecology, vol. 89(1), 2008, pp 280-288.

[4] A. R. Akhmetzhanov, F. Grognard and L. Mailleret, Long term preypredator dynamics induced by short term optimal predatory behavior, In preparation

[5] A. Friedman, Differential Games, Wiley-Interscience; 1971.

[6] F. Hamelin and P. Bernhard, Uncoupling Isaacs equations in twoplayer nonzero-sum differential games. Parental conflict over care as an example, Automatica, vol. 44(3), 2008, pp 882-885.

[7] P. Cardaliaguet and S. Plaskacz, Existence and uniqueness of a Nash equilibrium feedback for a simple non-zero-sum differential game, International Journal of Game Theory, vol. 32(1), 2003, pp 33-71.

[8] A. Bressan and W. Shen, Semi-cooperative strategies for differential games, International Journal of Game Theory, vol. 32(4), 2004, pp 561-593.

[9] A.A. Melikyan, Generalized characteristics of first order PDEs: applications in optimal control and differential games, Birhäuser, Boston; 1998. 

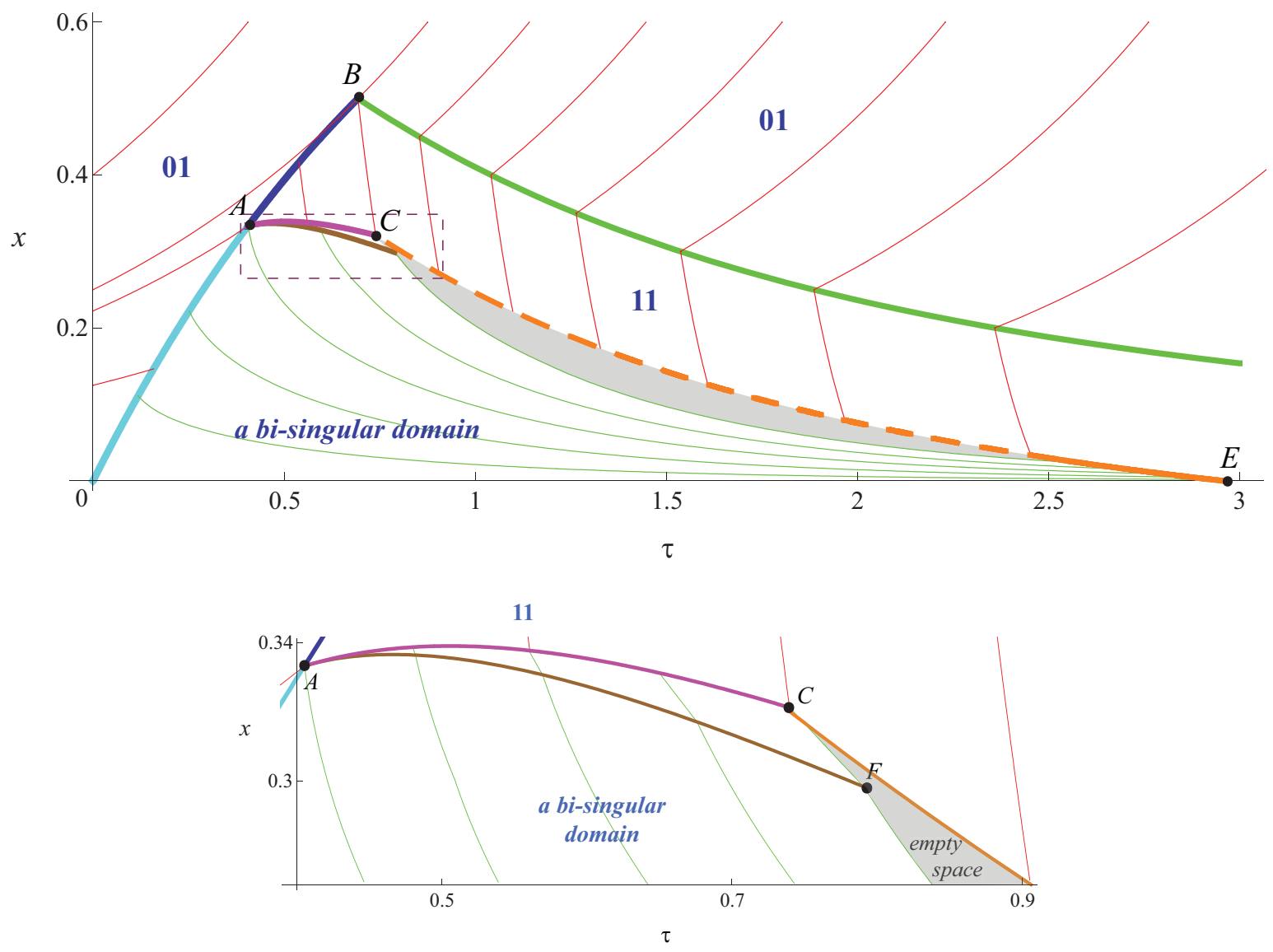

Fig. 10. Real game trajectories recovered from the bi-singular solution 